\title{
HYDRODYNAMIC INTERACTIONS IN MULTIPLE BODY ARRAY: A SIMPLE AND FAST APPROACH COUPLING BOUNDARY ELEMENT METHOD AND PLANE WAVE APPROXIMATION
}

\author{
Jitendra Singh \\ LUNAM Université \\ Ecole Centrale de Nantes-CNRS \\ 1 rue de la Noe \\ 44300 Nantes Cedex 3, France \\ Email: jitendra.singh@ec-nantes.fr
}

\author{
Aurélien Babarit \\ LUNAM Université \\ Ecole Centrale de Nantes-CNRS \\ 1 rue de la Noe \\ 44300 Nantes Cedex 3, France \\ aurelien.babarit@ec-nantes.fr
}

\section{ABSTRACT}

The hydrodynamic forces acting on an isolated body could be considerably different than those when it is considered in an array of multiple bodies, due to wave interactions among them. In this context, we present in this paper a numerical approach based on the linear potential flow theory to solve full hydrodynamic interaction problem in a multiple body array. In contrast to the previous approaches that considered all bodies in an array as a single unit, the present approach relies on solving for an isolated body. The interactions among the bodies are then taken into account via plane wave approximation in an iterative manner. The boundary value problem corresponding to a isolated body is solved by the Boundary Element Method (BEM). The approach is useful when the bodies are sufficiently distant from each other, at-least greater than five times the characteristic dimensions of the body. This is a valid assumption for wave energy converter devices array of point absorber type, which is our target application at a later stage. The main advantage of the proposed approach is that the computational time requirement is significantly less than the commonly used direct BEM. The time savings can be realized for even small arrays consisting of four bodies. Another advantage is that the computer memory requirements are also significantly smaller compared to the direct BEM, allowing us to consider large arrays. The numerical results for hydrodynamic interaction problem in two arrays consisting of 25 cylinders and same number of rectangular flaps are presented to validate the proposed approach.

\section{Introduction}

The motivation of this study originates from the need of modeling large arrays of wave energy converter devices (WECs) of point absorber type. Installation of several WECs would be required to extract reasonable amount of energy, and the wave interaction effects may become necessary to take into account in order to make reliable estimates on the energy production for a particular array configuration. In practice, due to mooring and maintenance issues for example, the separating distance between the devices could be a few hundred meters [1]. This study aims to deal with hydrodynamic interaction problem in such configurations of the bodies.

The classical approach to solve full hydrodynamic in- 
teraction problem is to model the problem in the linear potential theory framework. Essentially the diffraction and radiation problems in frequency domain are solved, and the hydrodynamic coefficients: excitation force, added mass and wave damping, can then be easily post-processed. A judicious choice to solve these problems is by the Boundary Element Method (BEM), since the discretization of the boundary alone is required. However, when array consists of several bodies, the discretization element increases, and use of the BEM becomes prohibitive due to computational requirements of solving a dense linear matrix system. In addition, the solution of diffraction and radiation problems are sought for a wide range of frequencies to perform comprehensive hydrodynamic analysis, thereby becoming more difficult to do in reasonable time. There have been attempts to accelerate the BEM by coupling with the fast methods, such as fast multipole methods (FMM) [2] and fast Fourier transforms (FFT) [3]. In wave hydrodynamics field the BEM-FMM coupling to solve diffraction and radiation problems has been realized in [4-6] for specific applications. The acceleration using fast Fourier transforms has been performed in [7] and others. However, these approaches have some limitations. In FMM, the existing multipole series expansion of the free surface Greens function is conditionally convergent, thereby restricting scope of developments for generic three-dimensional bodies. Whereas, in FFT methods, construction of a grid and projection operations in full domain of the sparsely placed body array would lead to a sub-optimal algorithm.

Besides aforementioned fast methods, some interesting (semi-) algebraic approaches have also been proposed. An interaction approach based on multiple scattering theory [8] and combining direct matrix method is presented in [9]. An extension of this theory, so called hierarchical interaction theory, is presented in [10]. The plane wave approximation, also known as wide space approximation, coupled with direct matrix approach is proposed by Simon [11]. This approach is further improved to reduce the wide spacing requirement by taking non-plane correction in [12]. A detailed comparison of the plane wave approximation and the multiple scattering approach is performed on the arrays of five WEC devices in [13]. We may highlight that the aforementioned approaches are very interesting for axisymmetric bodies.

In this study, we propose a simple approach for hydrodynamic interactions in an array of large number of bod- ies of arbitrary shape, provided the bodies are sufficiently distant from each other. The approach couples efficiency of the BEM and simplicity of the plane wave approximation [11]. The central idea is that when the body are sufficient distant in an array then the effects of wave field emanating from one body can be taken into account at the other body as an additional plane incident wave. Here we use this approximation efficiently and develop a fast algorithm. The computational time and memory requirements using the proposed approach are significantly less than the direct BEM, and is applicable to bodies of arbitrary shape. Furthermore, the approach is simple and can be easily implemented in existing diffraction/radiation solvers.

\section{Problem statement}

We consider the problem of water waves incident on an assembly of $N$ floating three-dimensional bodies under the framework of linearized potential flow theory. Specifically, the flow is assumed to be invisicid, incompressible and irrotational, and the wave amplitude is small relative to the wavelength. With these assumptions the problem can be formulated in terms of a velocity potential $\Phi$ satisfying the Laplace's equation in the fluid domain and appropriate boundary conditions. For simplicity we consider that the fluid domain is of infinite depth and unbounded in horizontal directions. The motion is time harmonic with radian frequency $\omega$, i.e. $\Phi(x, y, z, t)=\operatorname{Re}\left\{\phi(x, y, z) e^{-i \omega t}\right\}$. The time dependence term is omitted hereafter.

The linearization permits writing the velocity potential $\phi$ as sum of the incident potential $\phi_{i n}$, the diffraction potential $\phi_{d}$ and the radiation potential $\phi_{r}$. The explicit form of the incident potential is given by

$$
\phi_{\text {in }}=\frac{g A}{\omega} e^{k z} e^{i k(x \cos \beta+y \sin \beta)}
$$

where $k=\omega^{2} / g$ is the wave number, $g$ is the acceleration due to gravity, $A$ is the amplitude and $\beta$ is the angle between the direction of propagation of the incident wave and the positive $x$-axis. The diffraction potential corresponds to the potential generated in response to the incident waves. The radiation potential represents the fluid disturbance due to the motions of the bodies in still water corresponding to six degree of freedom: surge, sway, heave, roll, pitch and yaw. 
The diffraction and radiation boundary value problems (BVPs) can be summarized as follows:

Diffraction problem

$$
\left\{\begin{array}{cc}
\Delta \phi_{d}=0 & \text { in fluid domain } \\
\nabla \phi_{d} \rightarrow 0 & z \rightarrow \infty \\
\frac{\partial \phi_{d}}{\partial z}-k \phi_{d}=0 & \text { at mean free surface position } z=0 \\
\frac{\partial \phi_{d}}{\partial n}=-\frac{\partial \phi_{i n}}{\partial n} & \text { on mean wetted body surface }
\end{array}\right.
$$

Radiation problems

$$
\left\{\begin{array}{cc}
\Delta \phi_{r_{i}}^{j}=0 & \text { in fluid domain } \\
\nabla \phi_{r_{i}}^{j} \rightarrow 0 & z \rightarrow \infty \\
\frac{\partial \phi_{r_{i}}^{j}}{\partial z}-k \phi_{r_{i}}^{j}=0 & \text { at mean free surface position } z=0 \\
\frac{\partial \phi_{r_{i}}^{j}}{\partial n}=n_{i}^{j} & \text { on mean wetted body surface }
\end{array}\right.
$$

where indices $i$ and $j$ correspond to the degree of freedom of motion $(i=1 . .6)$ and the numeration of the body $(j=1 . . N)$, respectively. With this convention $n_{i}^{j}$ denotes the generalized normal vector on body $j$ with six components. The first three components of the generalized normal vector on body $j$ are the components of the unit normal vector $\mathbf{n}$ considered pointing outwards to $j^{\text {th }}$ body surface, and next three are the rotations $\mathbf{x} \times \mathbf{n}$ referred to the origin $\mathbf{x}=0$, while on all other bodies except $j$ all the components are zero.

Having solved the diffraction and radiation problems, it is straightforward to compute the hydrodynamic coefficients: excitation force, added mass and wave damping coefficients.

\subsection{Boundary integral equation formulation}

The BVPs (2) and (3) can be transformed into boundary integral equations by either using Green's identity involving potentials only (direct formulation), or involving particular source distribution (indirect formulation). We prefer the source formulation because both diffraction and radiation problems can be formulated in a common framework of Fredholm equation of second kind and implemented in our homemade software AQUAPLUS [14]. First the following integral equations is solved for the unknown source distribution $\sigma$ on the body surfaces $S=\bigcup_{k=1}^{N} S_{k}$

$$
\frac{\sigma(\mathbf{x})}{2}-\frac{1}{4 \pi} \int_{S} \sigma\left(\mathbf{x}^{\prime}\right) \frac{\partial G\left(\mathbf{x}^{\prime}, \mathbf{x}\right)}{\partial n_{\mathbf{x}}} d S\left(\mathbf{x}^{\prime}\right)=\left\{\begin{array}{c}
-\frac{\partial \phi_{\text {in }}}{\partial n}(\mathbf{x}) \\
n_{i}^{j}(\mathbf{x})
\end{array},\right.
$$

where $\mathbf{x}^{\prime}$ and $\mathbf{x}$ are the source and field point, respectively. The function $G\left(\mathbf{x}^{\prime}, \mathbf{x}\right)$ is the free surface Green function taking into account the linearized free surface conditions, conditions at the sea bottom and radiation conditions at infinity [15]. Having solved (4) for the density $\sigma$, the velocity potential can be computed at any arbitrary point by the following equation:

$$
\phi(\mathbf{x})=-\frac{1}{4 \pi} \int_{S} \sigma\left(\mathbf{x}^{\prime}\right) G\left(\mathbf{x}^{\prime}, \mathbf{x}\right) d S\left(\mathbf{x}^{\prime}\right)
$$

\section{BEM coupling with plane wave approximation}

Let us point out that the discretization of (4) leads to a dense matrix system. For an array consisting of $N$ bodies, the number of diffraction and radiation problems to be solved for motion in one degree of freedom are $(1+N) n_{f}$, where $n_{f}$ is the number of the frequencies. When $N$ is large it is not feasible through direct BEM to solves the problems in reasonable time. Keeping this in focus, we now describe a simple and fast approach to solve the BVPs through coupling BEM and plane wave approximation. Specifically we show how to take into account multiple interactions through plane wave approximation when bodies are sufficiently well separated.

For simplicity, we first consider the interaction phenomenon in an array of two bodies. Due to incident waves (diffraction problem) or independent motion (radiation problem) the body 1 scatter waves that excite body 2 . The body 2 responds to this excitation and in turn send outgoing waves towards body 1 which also respond to this excitation and so on. In the same vain, the body 2 interacts with body 1 due to incident waves or independent motion of the former. In what follows, we will approximate the impact of outgoing waves from a body by incident plane wave potential on all other bodies. This can be understood from the fact, as outlined by Simon [11], that far from the generating body the outgoing waves have small curvature and they act as plane waves on the incident body. Further, as the local wave field decay with distance, we can expect that 
it will not influence other bodies. However, for numerical accuracy the criterion 'sufficiently far' require careful investigation. On the basis of our numerical experiments we can point out that when the separating distance between the bodies is greater than approximately five times the characteristic dimensions of the body the approximation provides reliable results. The algorithm based on the above idea is formulated for $N$ body array in three steps which are described below.

Step 1: Contributions of individual bodies

In this step we compute the potential on each body by considering them isolated, i.e. hypothetically assuming bodies have no interaction with each other. This is straightforward to compute by solving integral equations (4 and 5) on the wetting body surface of individual body. The size of linear system to be solved is equal to the number of panels of a single body. In the first iteration the usual boundary conditions in (2 and 3) for diffraction and radiation problems are assigned locally to a body. The boundary conditions for the subsequent iterations are obtained in step 2 taking into account wave interactions. We can point out an additional advantage when bodies are of same shape. The influence matrices corresponding to Green function and its normal derivative matrices for a single body can be stored and used for all other bodies whenever their contribution are sought.

Step 2: Contributions due to wave interaction

As mentioned, we approximate the potential due to body $i$ on body $j$ by the potential of an incident plane wave. Since we know the source distribution on an arbitrary body $i$ from step 1, the potential at the mean center position $\left(x_{j}, y_{j}, 0\right)$ is computed for all other bodies using (5); denoted by $A_{i j}$. The total effect at body $j$ is taken as the sum of the effects from all other bodies in terms of incident plane waves of complex amplitude $A_{i j}$, i.e.

$$
\phi_{j}^{*}=\sum_{i=1}^{N} A_{i j} e^{k z} e^{i k\left(\left(x-x_{j}\right) \cos \theta_{i j}+\left(y-y_{j}\right) \sin \theta_{i j}\right)}, j \neq i
$$

where $\theta_{i j}$ is the angle subtended by mean center position of body $i$ while considering origin at the mean center position of body $j$. Having computed the effect at all the bodies in the array we compute the contribution of all the bodies as isolated (step 1) due to the excitation induced by $\phi^{*}$.

Step 3: Check for convergence

At each iteration the step 1 and 2 contributes towards the total potential on the body. This process is repeated till the amplitude of the incident waves $\left|A_{i j}\right|$ becomes smaller than $10^{-3}$ for all the bodies or maximum number of iterations are reached. The maximum number of iterations are set as twice the number of bodies in the array. According to our numerical investigation for a few frequencies the number of iterations are higher than the number of bodies, but for the majority the number of iterations is much smaller.

\section{Numerical examples}

We first consider hydrodynamic interaction problem in two arrays consisting of 25 floating rectangular flaps and same number of cylinders for accuracy check. The numerical results obtained by the proposed approach are compared with the direct BEM using constant panels. Next, some results of accuracy dependency on separating distance between the bodies and comparison of computational time between the direct BEM and the proposed approach as a function of number of bodies, are provided.

\subsection{Floating rectangular flaps}

The flaps are arranged in a regular triangular pattern, as shown in figure (1). The width and draught of each flap is 10 meter $(m)$ and length $5 m$. Each rectangular flap is discretized with 504 panels. The direction of propagation of the incident waves is $\beta=0$. The amplitude of surge and sway exciting forces on arbitrary selected flaps are shown in figures (2) and (3). The results from the proposed approach are plotted in lines and those by direct BEM are marked by $(+)$ in all cases. For radiation forces the notation $[m, n ; p, q]$ is used to indicate the force on body $p$ in direction $q$ due to the forced oscillations of body $m$ in direction $n$. Some results of added mass and wave damping coefficients are plotted in figures (4) and (5). The number of iterations to attain convergence for diffraction problem is shown in figure (6) (red curve). A similar convergence pattern is observed for radiation problems. However, there is a significant difference in computation time of 

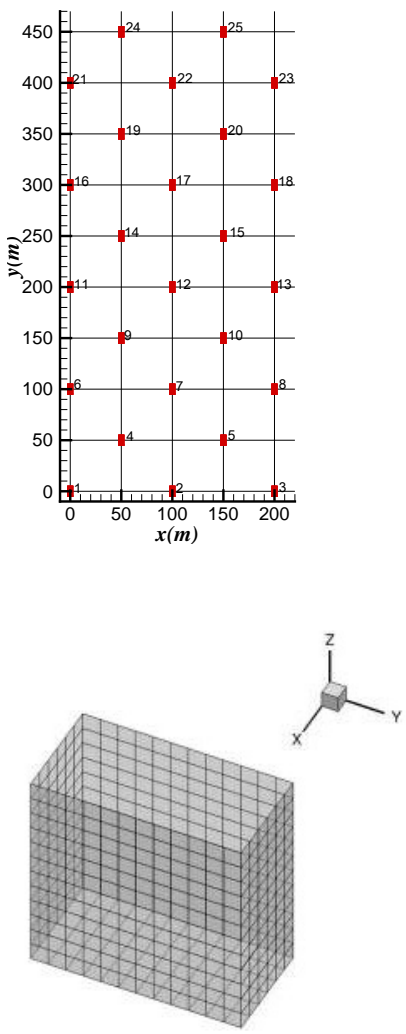

FIGURE 1. Array layout and surface mesh representation of rectangular flap

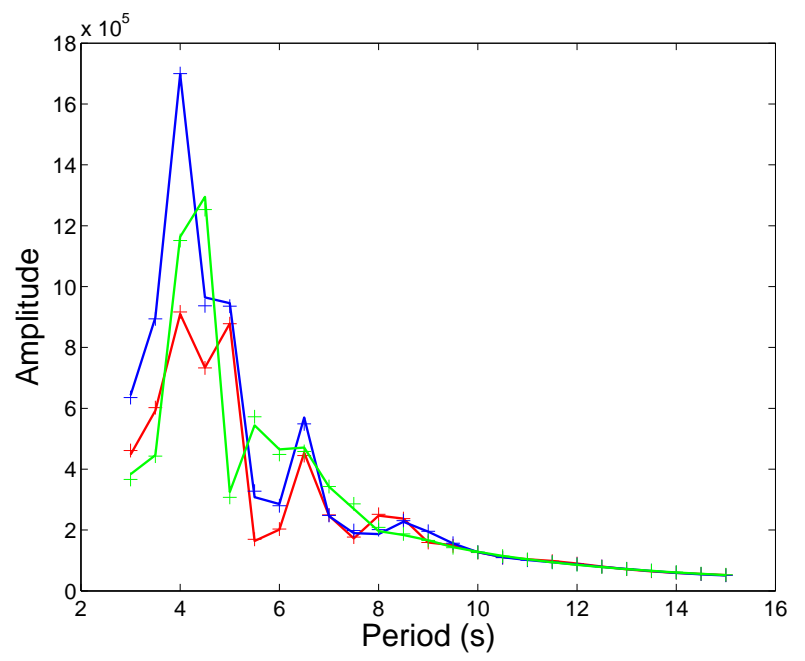

FIGURE 2. Surge exciting forces $(\mathrm{kN} / \mathrm{m})$ on barges 1-red, 7blue, 13-green.

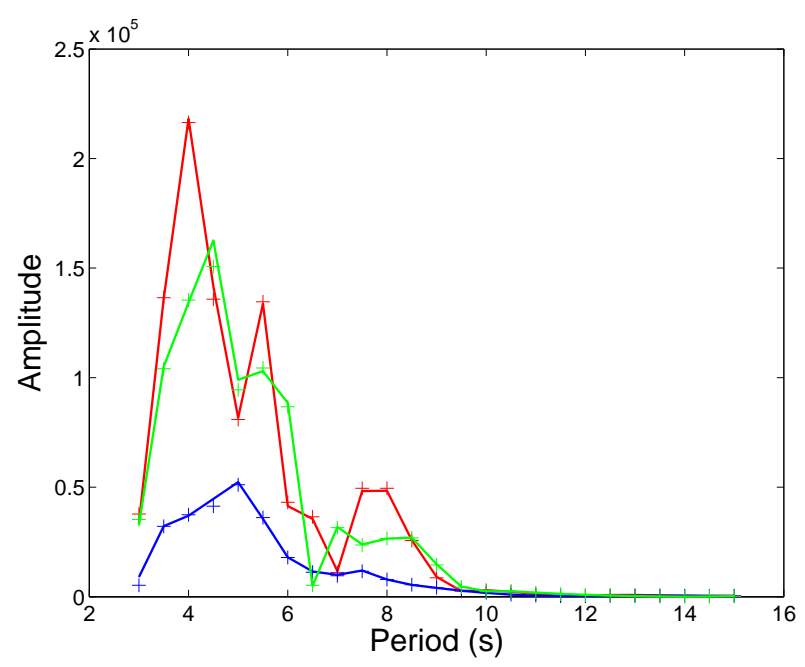

FIGURE 3. Sway exciting forces $(\mathrm{kN} / \mathrm{m})$ on barges 5-red, 12blue, 19-green.

both the approaches. Solving diffraction or radiation BVP for 25 frequencies through direct BEM took more than 8 hours, whereas the proposed approach took approximately 12 minutes.

\subsection{Floating cylinders}

In this array the cylinders are arranged in a regular square pattern, shown in figure (7). Each cylinder has diameter and draft of $10 \mathrm{~m}$ and is discretized with 560 panels. The direction of propagation of the incident waves is $\beta=0$. The excitation forces for arbitrarily selected bodies in surge, sway and heave motion computed by the proposed approach and direct BEM are shown in figures (8), (9), (10), respectively. The added mass and damping coefficients are shown in figures (11) and (12). It can be noticed that the results for this array configuration are more accurate than those of the first array consisting of rectangular flaps. This is because the bodies are more distantly placed in cylindrical array in comparison to rectangular flap array. In this example also there is significant difference in the computational time by the proposed approach and the direct BEM approach. The presented approach took less than 15 minutes, whereas the direct BEM took more than 10 hours. The number of iterations to attain convergence for diffraction problem is shown in figure 6 (blue curve) and the pattern is similar to the previous example.

We now investigate the dependence of separating dis- 

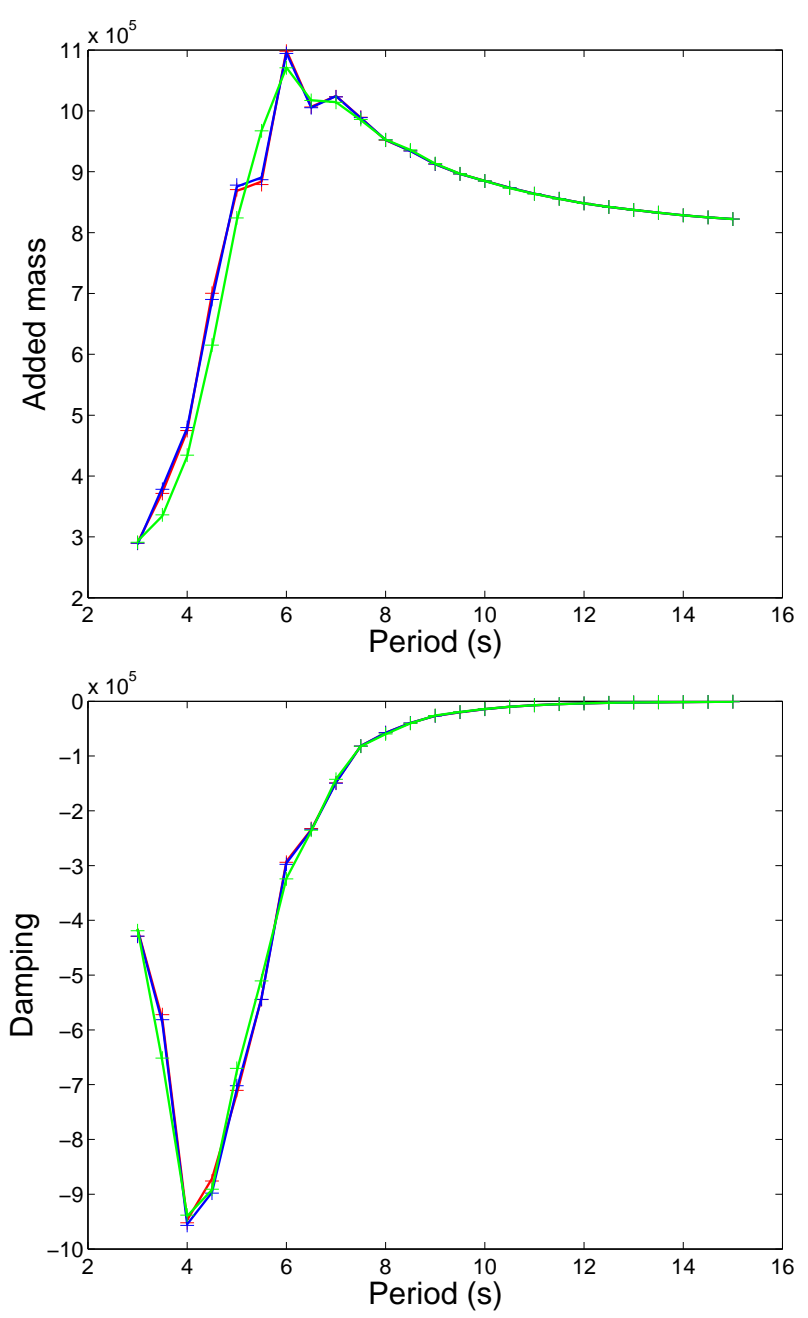

FIGURE 4. Added mass $\left(k N / m . s^{2}\right)$ and wave damping $(\mathrm{kN} / \mathrm{m} . \mathrm{s})$ coefficients $[10,1 ; 10,1]$-red, [20,1;20,1]-blue, $[23,1 ; 23,1]$-green.

tance between the bodies on overall accuracy of the computations. For this we consider an array consisting of 9 cylinders arranged in a square array with separating distance ranging from 25 to 125 meters. The dimensions of the cylinders are same as in previous example. The relative errors in excitation forces, i.e

$$
\text { Error }=\frac{\left|F_{e x}^{\text {direct }}-F_{e x}^{\text {present }}\right|}{\left|F_{e x}^{\text {direct }}\right|},
$$

in surge and heave motion on the cylinder positioned at the center of the array are plotted in figure (13). The error is
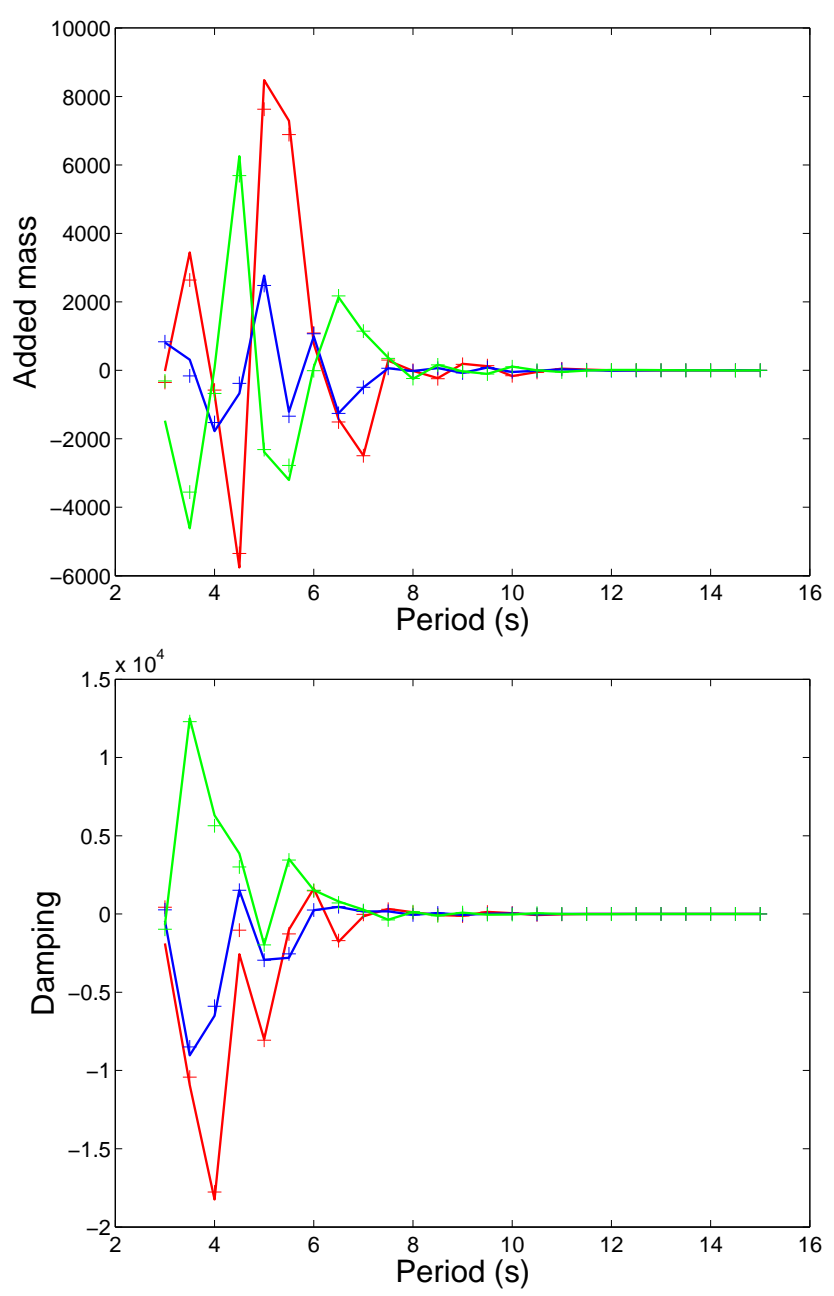

FIGURE 5. Added mass $\left(k N / m . s^{2}\right)$ and wave damping $(k N / m . s)$ coefficients $[5,2 ; 5,1]$-red, $[10,2 ; 10,1]$-blue, $[20,2 ; 20,1]$-green.

less than 5\% when the separating distance is greater than five times the dimensions of the cylinder. As expected, the error decrease as the separating distance increase.

Further to show that the approach can be useful for bigger arrays, we assess the computational time by the present approach as a function of number of bodies. For simplicity, the layout of the cylinders is again in a square array. The comparison of computational time using present approach and direct BEM is shown in figure (14). The time corresponding to direct BEM represents CPU time to solve for one period using direct Gauss solver. Whereas for the presented approach we plot average time for 12 periods 


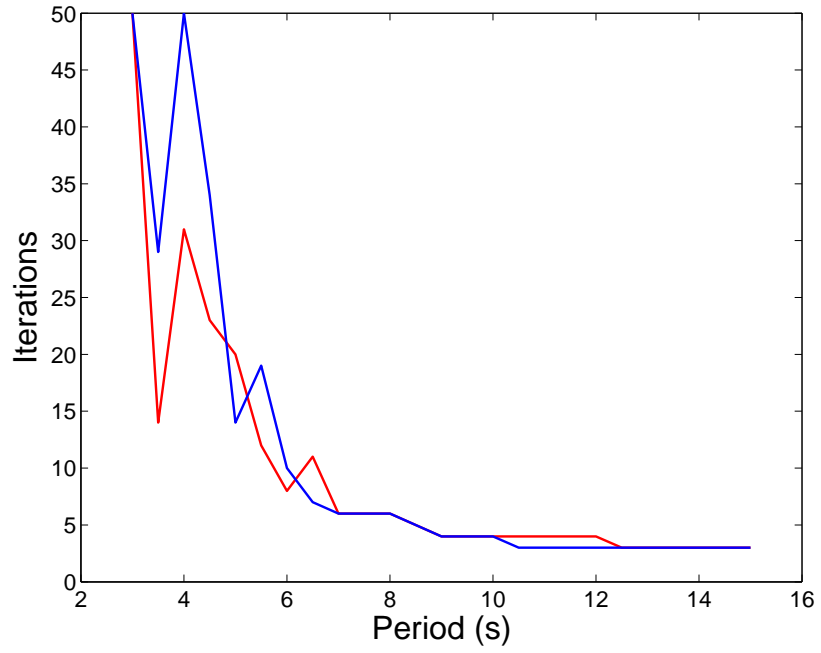

FIGURE 6. Convergence pattern for diffraction problems
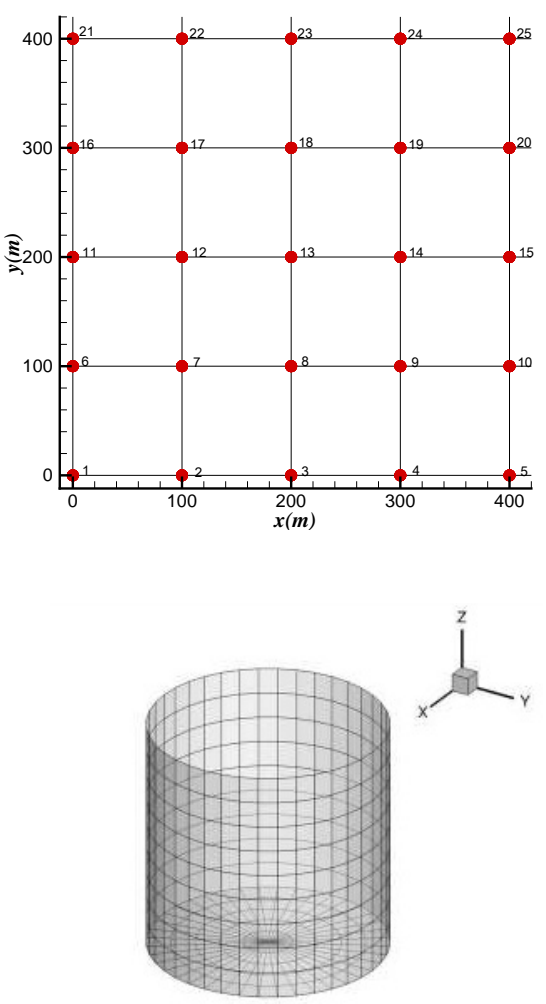

FIGURE 7. Array layout and surface mesh representation of cylindrical body

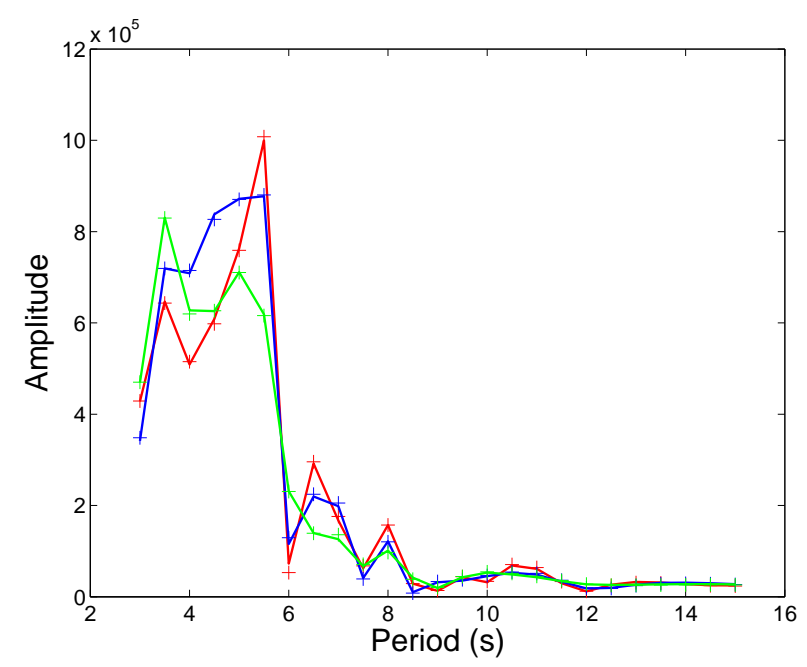

FIGURE 8. Excitation forces in surge $(\mathrm{kN} / \mathrm{m})$ on cylinders 7red, 13-blue, 19-green.

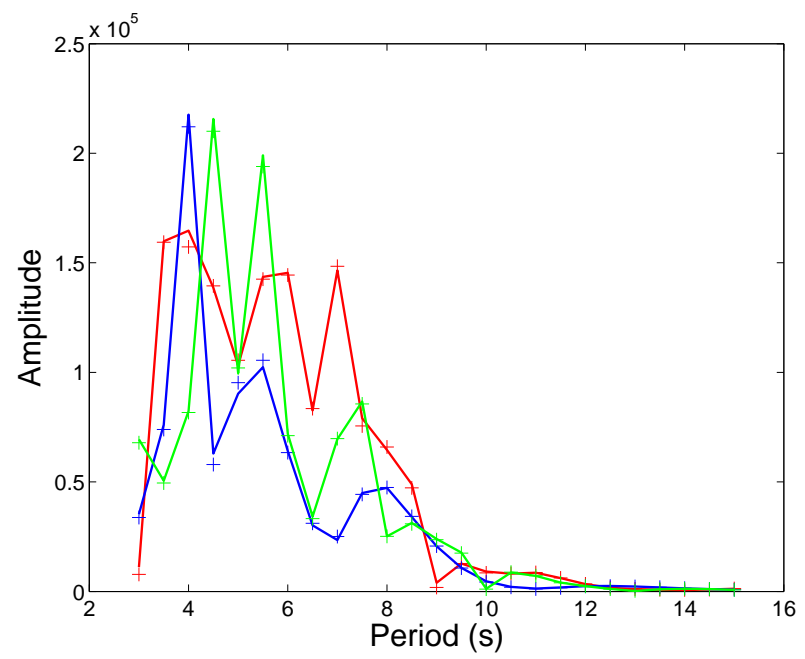

FIGURE 9. Excitation forces in sway $(\mathrm{kN} / \mathrm{m})$ on cylinders 3red, 9-blue, 16-green.

ranging from 3 to 14 seconds. This is because the number of iterations required to satisfy the convergence criterion depends on the period, as shown in figure (6). Figure (14) suggests that we can solve full hydrodynamic interaction problem in bigger arrays in reasonable time, which is clearly not feasible by direct BEM. We may point out that the memory requirements using present approach are less than the direct BEM even when we store the influence coefficients corresponding to a single body. 


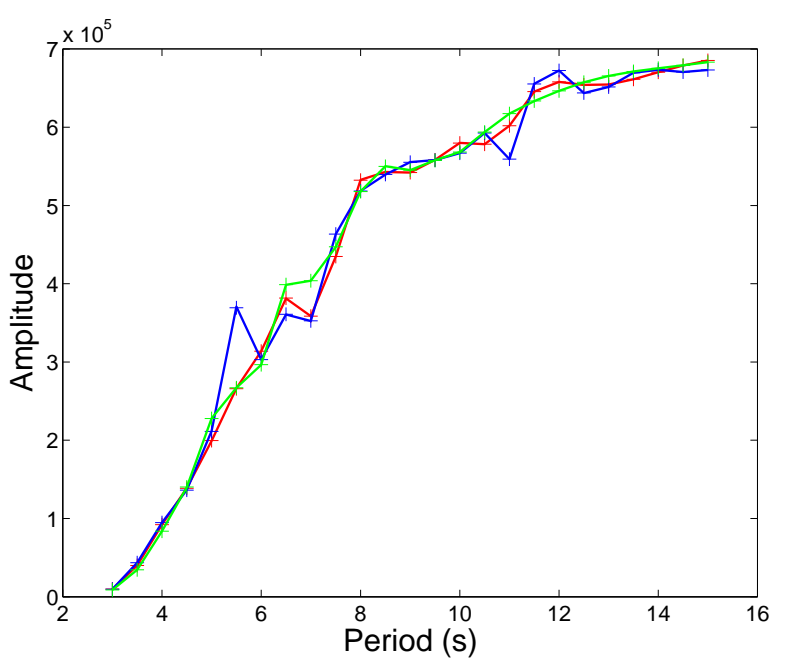

FIGURE 10. Excitation forces in heave $(\mathrm{kN} / \mathrm{m})$ on cylinders 2-red, 11-blue, 24-green.

\section{Summary and outlook}

We have proposed a simple approach to solve the hydrodynamic interaction in an array of multiple bodies by coupling BEM and plane wave approximation. Instead of considering an array as a single unit, the approach solves for an isolated body in an iterative manner. The interactions are taken into account by plane wave approximation. The approach gives good results when the bodies are separated by approximately more than five times the dimensions of the body. Numerical results on different array configuration shows that the proposed method provide viable results in comparison to the direct BEM. Most importantly, the approach is efficient in terms of computational time and memory as well as easy to implement. In fact very less efforts are required to modify the existing diffraction/radiation solvers. The approach now makes it possible to make parametric studies and investigate the performance of large number of WEC devices in reasonable time, which is currently in progress. The other aspect of the development would be to consider the interactions due to local wave field through incorporating the evanescent and propagating modes in the formulation.

\section{Acknowledgements}

The authors gratefully acknowledge the financial support from the French environmental agency ADEME
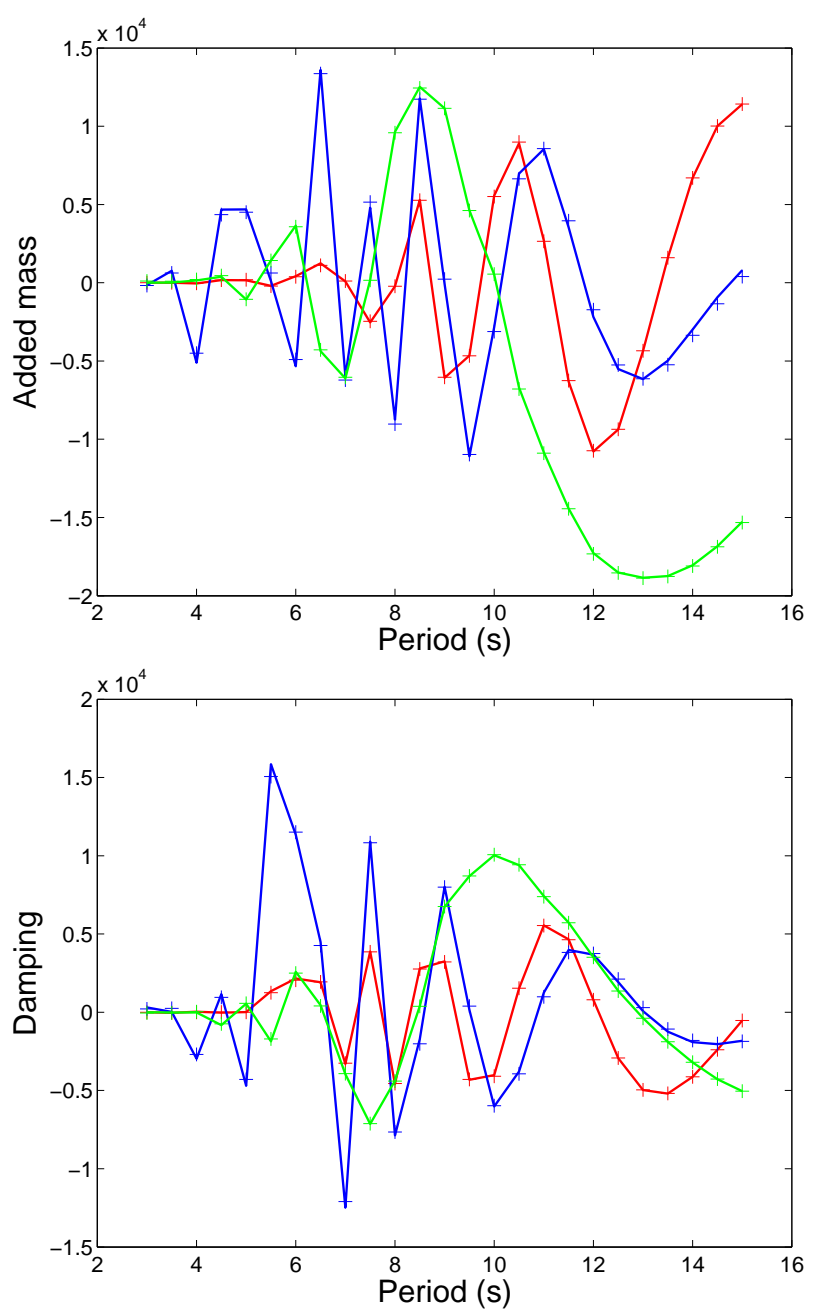

FIGURE 11. Added mass $\left(\mathrm{kN} / \mathrm{m} \cdot \mathrm{s}^{2}\right)$ and wave damping coefficients $(k N / m . s)[17,3 ; 15,3]$-red, [12,1;15,3]-blue, $[10,3 ; 15,3]$-green.

through the funding scheme "Démonstrateurs en énergies renouvelables et chimie verte" of Investissements d'avenir.

\section{REFERENCES}

[1] Borgarino, B., Babarit, A., and Ferrant, P., 2012. "Impact of wave interactions effects on energy absorption in large arrays of wave energy converters". Ocean Engineering, 41, pp. 79-88.

[2] Greengard, L., and Rokhlin, V., 1987. "A fast algorithm for particle simulations". Journal of Computational Physics, 73(2), pp. 325-348.

[3] Phillips, J., and White, J., 1997. "A precorrected-fft 

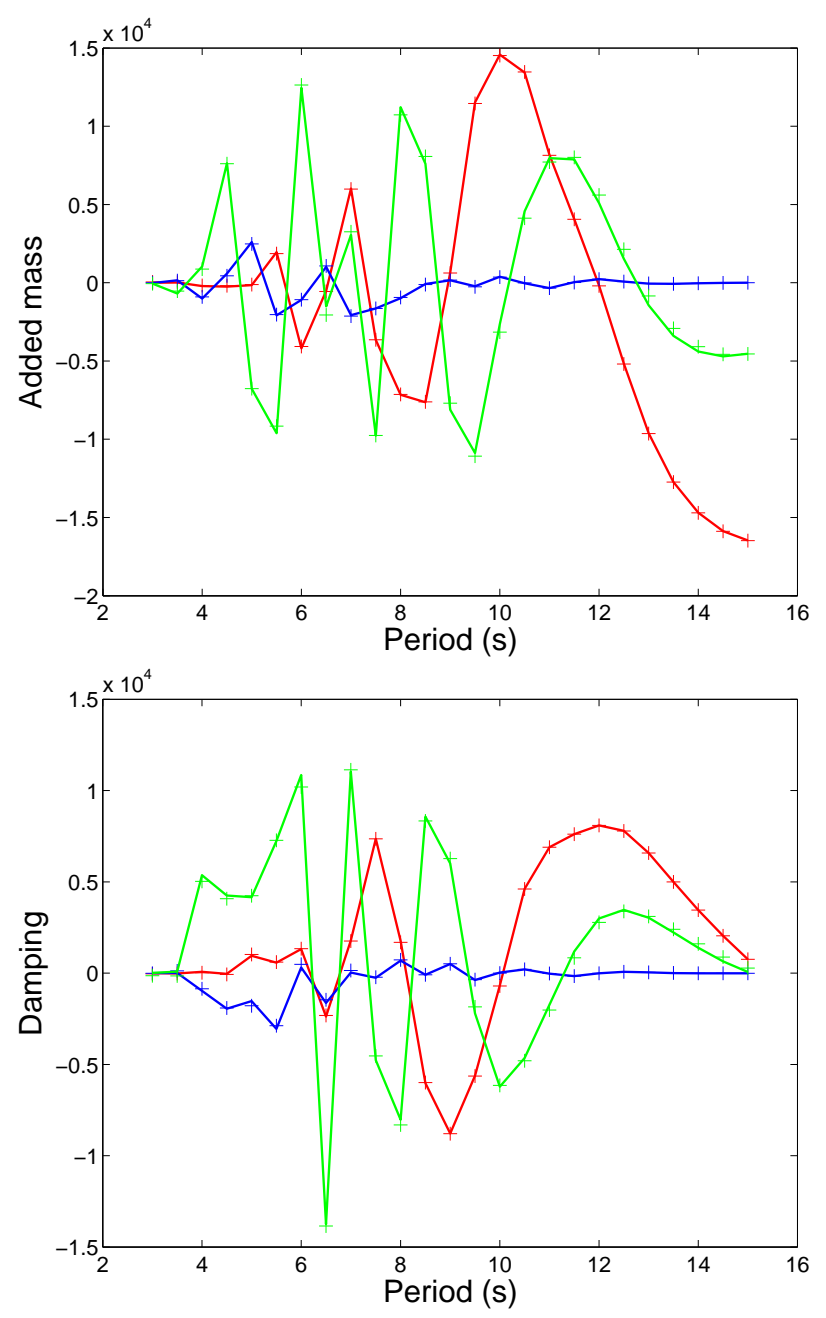

FIGURE 12. Added mass $\left(\mathrm{kN} / \mathrm{m} . \mathrm{s}^{2}\right)$ and wave damping coefficients $(k N / m . s)$ [2,3;6,3]-red, [8,2;6,3]-blue, [13,1;6,3]green.

method for electrostatic analysis of complicated 3-d structures". IEEE Transactions on Computer-Aided Design of Integrated Circuits and Systems, 16(10), pp. 1059-1072.

[4] Utsunomiya, T., and Watanabe, E., 2002. "Accelerated higher order boundary element method for wave diffraction/radiation problems and its applications". Proceedings of 12th International Offshore and Polar Engineering Conference, pp. 305-312.

[5] Teng, B., and Gou, Y., 2006. "Fast multipole expansion method and its application in bem for wave diffraction and radiation". Proceedings of 16th Inter-

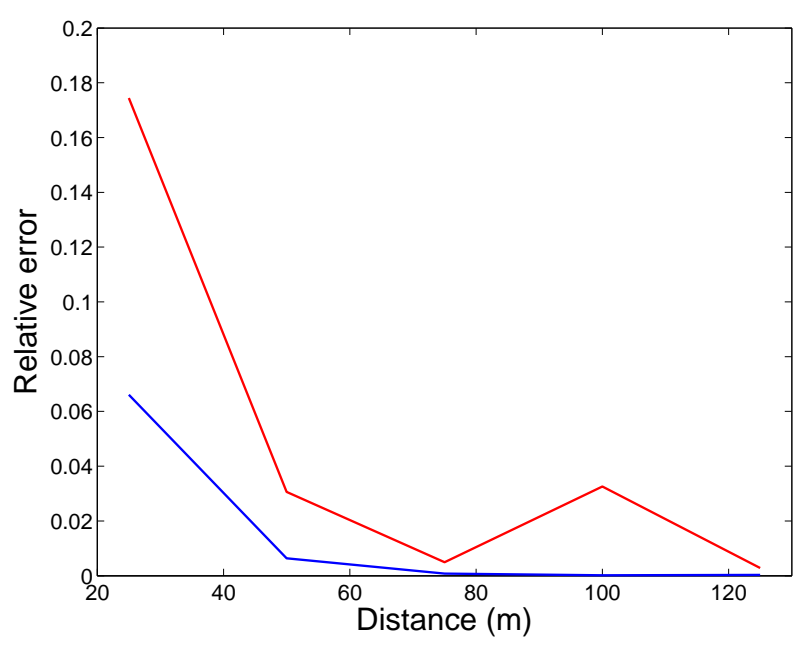

FIGURE 13. Error in surge (red curve) and heave (blue curve) exciting forces.

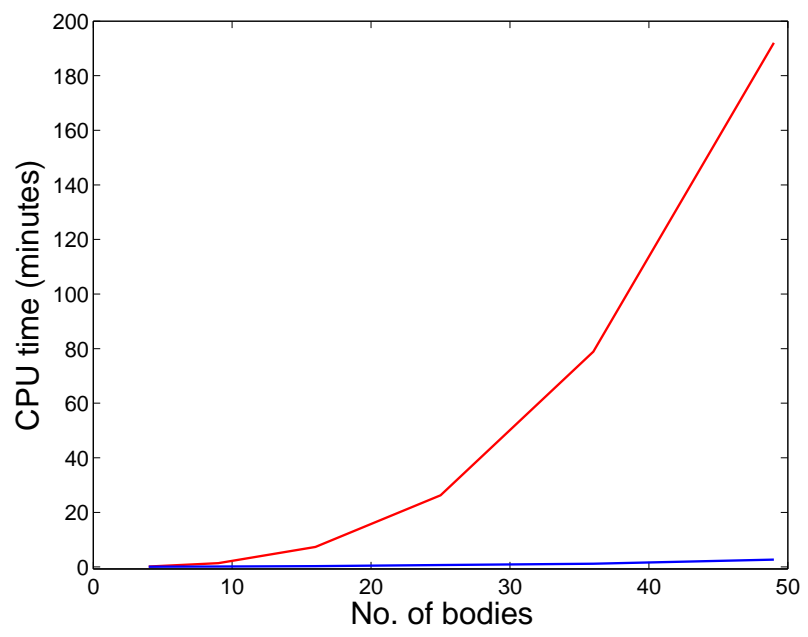

FIGURE 14. CPU time comparison of direct BEM (red curve) with present approach (blue curve).

national Offshore and Polar Engineering Conference, pp. 318-325.

[6] Borgarino, B., Babarit, A., and Ferrant, P., 2011. "An implementation of the fast multipole algorithm for wave interaction problems on sparse arrays of floating bodies". Journal of Engineering Mathematics, pp. 118.

[7] Kring, D., Korsmeyer, T., Singer, J., and White, J., 2000. "Analyzing mobile offshore bases using ac- 
celerated boundary-element methods". Marine Structures, 13(4), pp. 301-313.

[8] Ohkusu, M., 1974. "Hydrodynamic forces on multiple cylinders in waves". In International Symposium on the Dynamics of Marine Vehicles and Structures in Waves, 1974.

[9] Kashiwagi, M., 2000. "Hydrodynamic interactions among a great number of columns supporting a very large flexible structure". Journal of Fluids and Structures, 14(7), pp. 1013-1034.

[10] Kagemoto, H., and Yue, D., 1986. "Interactions among multiple three-dimensional bodies in water waves: an exact algebraic method". Journal of Fluid Mechanics, 166(1), pp. 189-209.

[11] Simon, M., 1982. "Multiple scattering in arrays of axisymmetric wave-energy devices. pt. 1: a matrix method using a plane-wave approximation”. Journal of Fluid Mechanics, 120, pp. 1-25.

[12] McIver, P., and Evans, D., 1984. "Approximation of wave forces on cylinder arrays". Applied Ocean Research, 6(2), pp. 101-107.

[13] Mavrakos, S., and McIver, P., 1997. "Comparison of methods for computing hydrodynamic characteristics of arrays of wave power devices". Applied Ocean Research, 19(5), pp. 283-291.

[14] Delhommeau, G., 1993. "Seakeeping codes aquadyn and aquaplus". 19th WEGMENT School, Numerical simulation of hydrodynamics: ship and offshore structures.

[15] Newman, J., 1985. "Algorithms for the free-surface Green function". Journal of Engineering Mathematics, 19(1), pp. 57-67. 